\title{
Improvement of classification accuracy in a phase-tagged steady-state visual evoked potential-based brain computer interface using multiclass support vector machine
}

\author{
Chia-Lung Yeh', Po-Lei Lee ${ }^{1,2,3^{*}}$, Wei-Ming Chen ${ }^{4}$, Chun-Yen Chang ${ }^{5}$, Yu-Te Wu ${ }^{6}$ and Gong-Yau Lan ${ }^{7 *}$
}

\author{
* Correspondence: pllee@ee.ncu. \\ edu.tw; jerrylan3812@gmail.com \\ ${ }^{1}$ Department of Electrical \\ Engineering, National Central \\ University, Jhongli, Taiwan \\ ${ }^{7}$ Department of Medical Image, \\ Cheng Hsin General Hospital, Taipei, \\ Taiwan \\ Full list of author information is \\ available at the end of the article
}

\begin{abstract}
Background: Brain computer interface $(\mathrm{BCl})$ is an emerging technology for paralyzed patients to communicate with external environments. Among current $\mathrm{BCls}$, the steady-state visual evoked potential (SSVEP)-based BCI has drawn great attention due to its characteristics of easy preparation, high information transfer rate (ITR), high accuracy, and low cost. However, electroencephalogram (EEG) signals are electrophysiological responses reflecting the underlying neural activities which are dependent upon subject's physiological states (e.g., emotion, attention, etc.) and usually variant among different individuals. The development of classification approaches to account for each individual's difference in SSVEP is needed but was seldom reported.

Methods: This paper presents a multiclass support vector machine (SVM)-based classification approach for gaze-target detections in a phase-tagged SSVEP-based BCI. In the training steps, the amplitude and phase features of SSVEP from off-line recordings were used to train a multiclass SVM for each subject. In the on-line application study, effective epochs which contained sufficient SSVEP information of gaze targets were first determined using Kolmogorov-Smirnov (K-S) test, and the amplitude and phase features of effective epochs were subsequently inputted to the multiclass SVM to recognize user's gaze targets.

Results: The on-line performance using the proposed approach has achieved high accuracy $(89.88 \pm 4.76 \%)$, fast responding time (effective epoch length $=1.13 \pm 0.02 \mathrm{~s}$ ), and the information transfer rate (ITR) was $50.91 \pm 8.70$ bits $/ \mathrm{min}$.

Conclusions: The multiclass SVM-based classification approach has been successfully implemented to improve the classification accuracy in a phase-tagged SSVEP-based $\mathrm{BCl}$. The present study has shown the multiclass SVM can be effectively adapted to each subject's SSVEPs to discriminate SSVEP phase information from gazing at different gazed targets.
\end{abstract}

\section{Background}

Brain computer interface (BCI) measures specific brain signals induced from elaborately designed tasks and translates the brain signals into control signals [1,2], which provides a promising channel for disabled patients to communicate with external environments. Among current noninvasive BCIs, the steady-state visual evoked potential

(c) 2013 Yeh et al.; licensee BioMed Central Ltd. This is an Open Access article distributed under the terms of the Creative Commons Attribution License (http://creativecommons.org/licenses/by/2.0), which permits unrestricted use, distribution, and reproduction in any medium, provided the original work is properly cited. 
(SSVEP)-based BCI has been widely mentioned due to its high information transfer rate (ITR) ( 70 bits/min), little training, and high accuracy [3,4]. SSVEP is sinusoidal-type electroencephalogram (EEG) signal generated by the human visual cortex that is synchronized and phase-locked to user's attended repetitive visual stimulation [5-7]. SSVEP-based BCIs place EEG electrodes in the vicinity of the occipital area and recognize user's gazed target by analyzing the frequency or phase characteristics of the measured SSVEPs.

SSVEP-based BCIs can be at least divided into two categories in terms of coding techniques, one is the frequency-coded SSVEP-based BCI and the other is the phasetagged SSVEP-based BCI [2,8-11]. Among current SSVEP-based BCIs, most SSVEPbased BCIs are frequency-coded systems. These frequency-coded systems utilize multifrequency flickers to induce subject's SSVEPs. Each visual target has a corresponding frequency which can be recognized on the estimated spectra, and the target number depends on how many frequencies are used for visual stimulation [8-10]. Nevertheless, due to the amplitude-frequency characteristic of SSVEP, some frequency ranges with poor SSVEP signal-to-noise ratio (SNR) should be excluded which usually results in a limited visual target number. In contrast, the phase-tagged system permits visual flickers to flash at the same frequency but being tagged with different phases. Accordingly, phase-tagged SSVEP systems have been developed to extend the available target number for SSVEP-based BCIs [2,4-12].

Several phase-tagged SSVEP-based BCIs have been developed. Lee et al. [2] implemented an eight-target system with flickering frequency set at $31.25 \mathrm{~Hz}$, and averaged over an amount of wave cycles (sixty cycles) to achieve 95\% accuracy. Jia et al. [12] developed a frequency and phase mixed coding technique, and found a phase mismatch between the phase difference of visual stimuli and the phase difference of measured SSVEP. Shyu et al. [13] designed a SSVEP-controlled hospital bed nursing system on FPGA platforms. Chang et al. [14] proposed stepping delay flickering sequence (SDFS) to achieve a phase-tagged SSVEP-based BCI independent of SSVEP phase calibration. Zhu et al. [4] accurately analyzed the phase synchrony between SSVEP and the flashing timing of visual stimulator measured from a photodiode. They concluded the variation of the phase difference between the flashing timing of visual stimulator and SSVEP, which might be caused by the phase deviations of visual stimulator. The variability is also called jitter, defined as the standard deviation of measured latencies [15]. Lopez-Gordo et al. [16] utilized phase-tagged amplitude modulation to drive four checkboard stimuli, and manifested the requisite of a calibration procedure for classifying the four targets. Though the SSVEP phase has been reported as an effective feature to implement SSVEP-based BCIs, nevertheless, the variation of the phase difference between visual stimuli and measured SSVEP, caused by the phase deviation of the visual stimulus [4,16,17], individual's emotional condition [18], selective attention [19], nicotine [20], anticipatory anxiety [21], etc., could cause deterioration in the detected accuracy. Therefore, an effective phase classification approach to account for each individual's phase difference of SSVEP is needed so that a reliable phase-tagged SSVEP-based BCI can be achieved.

This study presented a multiclass support vector machine (SVM)-based approach to cope with SSVEP phase variation in the use of a phase-tagged SSVEP-based BCI. SVM was originally introduced by Vapnik and its co-workers at AT\&T Bell Laboratories 
[22-25]. It has shown its transcendent performance in many applications [26], such as object identification [27], speaker identification [28], text categorization [29], etc. Input data in SVM is mapped into high-dimensional feature space and a hyperplane is determined to completely separate the input vectors into non-overlapping classes [30-33]. In this paper, the amplitude and phase features of SSVEPs, collected in every 4 cycles at $20 \mathrm{~Hz}$ flickering frequency from gazing at different phase-tagged flickers, were extracted to train the multiclass SVM classifier. The trained multiclass SVM was then utilized to discriminate subject's gazed targets. The multiclass SVM data classification using complex non-linear decision boundaries could be helpful to improve the statistical classification performance in phase-tagged SSVEP-based BCI design.

\section{Methods}

\section{A. Subjects and EEG preparation}

Twenty subjects (ten males and ten females), ages from 23 to 37 years old, were recruited to participate in this study. Each subject had corrected Snellen visual acuity of $6 / 6$ or better, with no history of clinical visual disease. Table 1 lists the demographic data of the participants. The research was carried out in compliance with Helsinki declaration. All subjects gave informed consent, and the study was approved by the Ethics Committee of Institutional Review Board (IRB), Taipei Veterans General Hospital, Taiwan. All measurements were noninvasive and the subjects were free to withdraw at any time without any penalty. One unipolar EEG channel was used by attaching an electrode $(\mathrm{Oz}(+))$ placed at $\mathrm{Oz}$ position with respect to a reference electrode $(\mathrm{Oz}(-))$ placed at the right mastoid. The ground electrode was placed in the frontal position (Fpz). These EEG electrode placements were based on the international EEG 10-20 system [34]. Oz EEG signals were amplified, pre-filtered within $0.1 \sim 100 \mathrm{~Hz}$ (PowerLabTM, ADInstrument, Castle Hill, NSW, Australia), and digitized at $1 \mathrm{KHz}$ (NI-USB 6259E, National Instrument) for further processes. The EEG recordings in all subjects were done by the same technician to minimize operation errors.

\section{B. System architecture and visual stimulus}

Four light-emitting diodes (LED) (part number: LYBSB93W1303R012BP, LedTech Electronics Co., Taiwan; rise time $<50 \mu$ s; wavelength ranging from 400 to $700 \mathrm{~nm}$ ), covered with thin white paper diffusers, were utilized as visual stimuli located on the four corners of a stimulus panel. A small cross was inlaid at the center of each visual stimulus to facilitate subject's eye fixation. All LEDs were flickering at $20 \mathrm{~Hz}$ achieved by alternative ON and OFF states, in which the luminance of ON and OFF states were 168.7 candelas $\left(\mathrm{cd} / \mathrm{m}^{2}\right)$ and $8.1 \mathrm{~cd} / \mathrm{m}^{2}$, respectively, measured by a luminance meter

Table 1 The demographic data of the participants in the multiclass SVM study

\begin{tabular}{ll}
\hline Number of subjects & $\mathbf{2 0}$ \\
\hline Gender (number; \%) & $10(50 \%)$ \\
Male & $10(50 \%)$ \\
$\quad$ Female & \\
Age (mean \pm SD; range) & $29.85 \pm 4.92(23-37)$ \\
$\quad$ Years & 2 \\
\hline
\end{tabular}


(LS-110; Konica Minolta Photo Imaging Inc., USA) resulting in Michelson contrast of 90.3\%. The four LEDs were tagged with distinct phases equally distributed over a full $360^{\circ}$ phase cycle. The designated phase for $i^{t h}$ LED is $\theta_{i}=(i-1) \times 90^{\circ}$ for $i=1 \sim 4$, with corresponding latency of

$$
t_{i}=\frac{\theta_{i}}{360^{\circ}} \times T
$$

where $\theta_{i}$ is the phase delay, $t_{i}$ is the latency for achieving $\theta_{i}, T=1 / f$, and $f=20 \mathrm{~Hz}$.

Figure 1a shows the system architecture of our phase-tagged SSVEP-based BCI. The stimulus panel was $60 \mathrm{~cm}$ in front of the participant. The four visual stimuli, tagged with $0^{\circ}, 90^{\circ}, 180^{\circ}$ and $270^{\circ}$, were labeled as ' $\mathrm{LED}_{1}$,' ' $\mathrm{LED}_{2}$,' $\mathrm{LED}_{3}$ ', and ' $\mathrm{LED}_{4}$ ', respectively. The flickering sequences for driving the four visual stimuli are shown in Figure 1b. The phase lags of $0^{\circ}, 90^{\circ}, 180^{\circ}$ and $270^{\circ}$ are generated by manipulated time delays of $0 \mathrm{~ms}, 12.5 \mathrm{~ms}$, $25 \mathrm{~ms}$ and $37.5 \mathrm{~ms}$, respectively, in the four flickering sequences (see Equation (1)). The

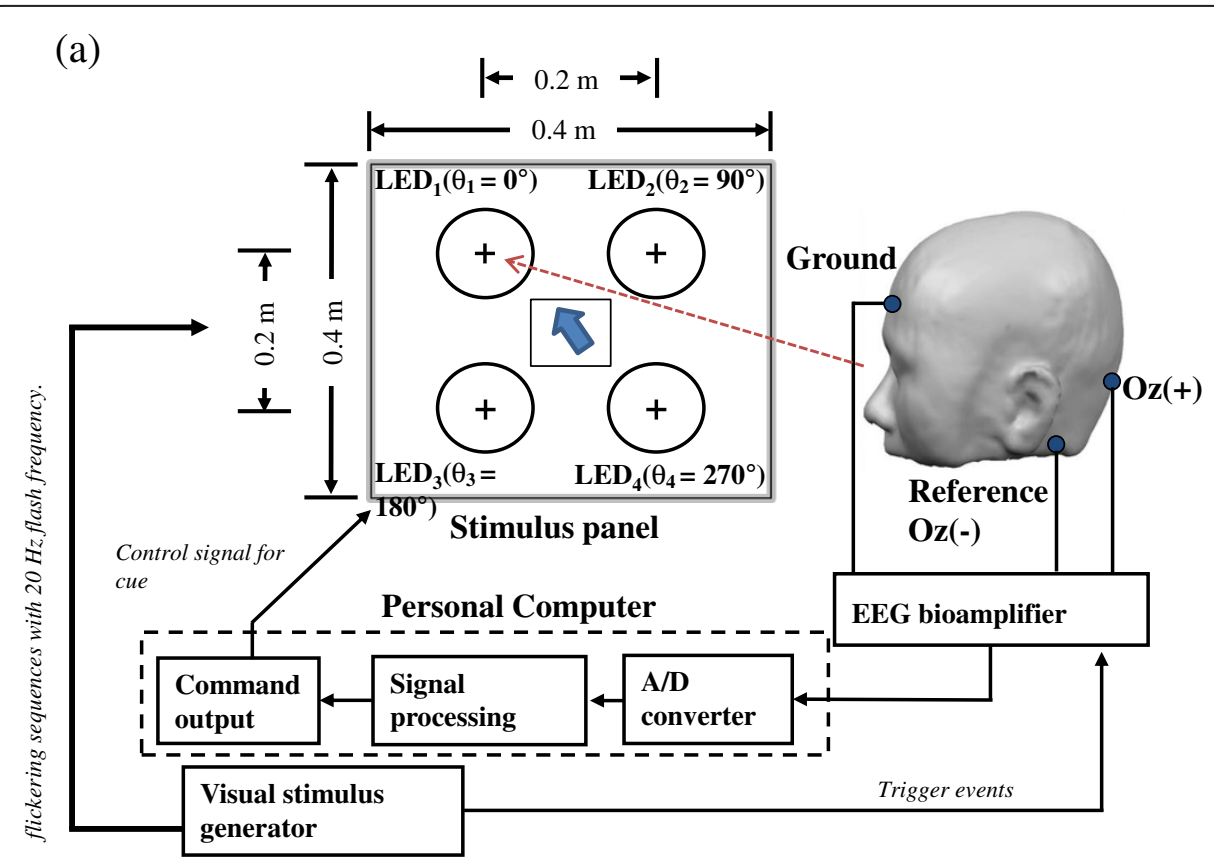

(b)

Microprocessor

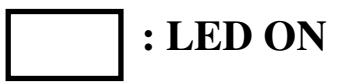

\section{: LED OFF}

Stimuli with

Trigger

\section{Trigger}

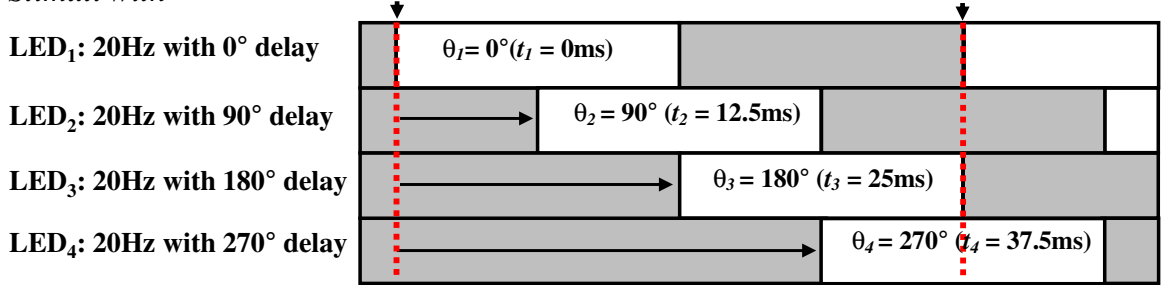

Figure 1 Block diagram and flickering sequences of our phase-tagged SSVEP-based BCI system. The system architecture of the system in (a). The four phase-tagged flickering sequences at $20 \mathrm{~Hz}$, where the inter-stimulus phase delay was set at $90^{\circ}\left( \pm 45^{\circ}\right.$ phase margin) in (b). 
flickering sequences were generated by a microprocessor (C8051F120, Silicon Laboratories Inc., USA), programmed with Keil IDE software. The flash onsets (from OFF states to ON states) in the flickering sequences served as trigger events for the subsequent process.

\section{Experimental tasks}

All subjects were requested to participate in a classification study and an application study. In the classification study, each subject was requested to gaze at each of the four visual stimuli for 60 seconds. After gazing at each visual stimulus, subjects were asked to take a $1 \mathrm{~min}$ rest. Another 60 seconds were also recorded from subject's resting state while keeping his/her eyes open. We took the first halves (30 seconds) of the five recordings (i.e., four gaze recordings and one eye-opened resting-state (non-gaze) recording) for multiclass SVM training, and the other halves of the five recordings were used to test the feasibility of multiclass SVM in classifying different conditions. Since accurate classification usually depends on correct information of input data, statistical Z-test was applied to the input vectors (see below) obtained from each gaze condition of training data and those input vectors which rejected the null hypothesis $(p<0.05)$ were excluded from multiclass SVM training. In the application study, the subjects were instructed to shift their eyes to target the four visual stimuli for 80 trials (20 trials for each visual stimulus) in randomized order. Each trial contained the following steps: (1) An onscreen message $\left(\nwarrow: \mathrm{LED}_{1} ; \nearrow: \mathrm{LED}_{2} ; \swarrow: \mathrm{LED}_{3} ; \searrow: \mathrm{LED}_{4}\right)$, located at the central position of the stimulus panel, was presented to instruct the subject to gaze at a designated visual stimulus; (2) The subject kept gazing at the designated visual stimulus until the subject received an auditory biofeedback. If the recognized gazed target was correct (i.e., the same as the one instructed by on-screen message), the auditory feedback would be a beep sound. Otherwise, a buzz sound would be generated to inform the subject of the wrong detection; (3) The subject perceived the auditory biofeedback, then shifted his/her gaze back to central position to prepare for next on-screen message. The experimental paradigm of the application study for each trial is shown in Figure 2.

\section{SSVEP signal processing}

\section{Extraction of SSVEP amplitude and phase features}

Oz EEG signals were bandpass-filtered within $17-23 \mathrm{~Hz}$ to obtain SSVEP responses by means of applying a causal Butterworth filter (6th-order, IIR Butterworth filter). The

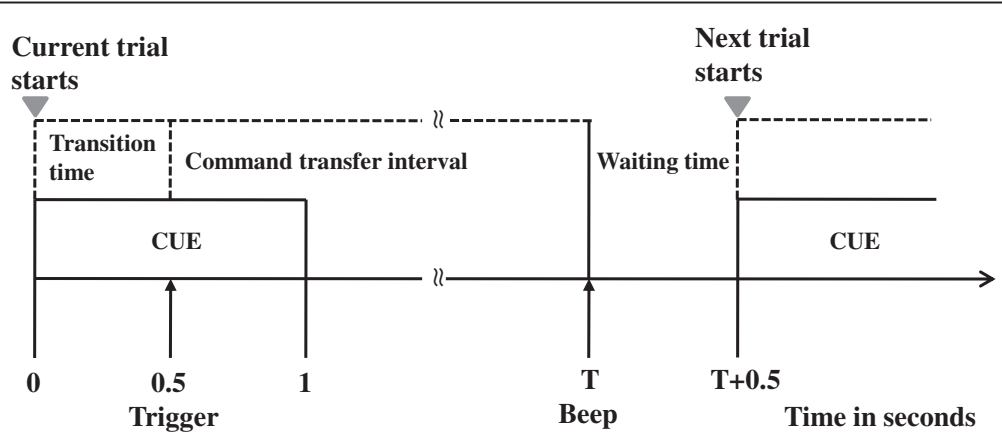

Figure 2 The experimental paradigm of the application study for each trial. 
frequency components of SSVEPs at $20 \mathrm{~Hz}$ were extracted in every 4 cycles using Fourier method with $75 \%$ overlapped sliding Hamming window. The frequency component at $i^{\text {th }}$ time window can be expressed as:

$$
F_{i}=\frac{1}{K} \sum_{n=1}^{K} s_{i}[n] \cdot e^{-j 2 \pi \frac{f_{0}}{f_{s}} n}
$$

in which $F_{i}$ is the frequency component at $i^{t h}$ time window, $s_{i}[n]$ is the bandpass filtered data of $i^{\text {th }}$ time window, $K(K=200)$ represents the sliding Hamming window length, and $f_{s}\left(f_{s}=1 \mathrm{KHz}\right)$ and $f_{O}\left(f_{o}=20 \mathrm{~Hz}\right)$ are the sampling frequency and visual stimulus flickering frequency. The amplitude and phase features of frequency component $F_{i}$ are arranged as an input vector, denoted as $\boldsymbol{x}_{i}$, subjected to input points of multiclass SVM classification. The input vector $\boldsymbol{x}_{i}$ is represented as

$$
x_{i}=\left[\begin{array}{c}
\left|F_{i}\right| \\
\left\llcorner\left(F_{i}\right)\right.
\end{array}\right] \text {, }
$$

where $|\cdot|$ is the absolute value operator, $\angle\left(F_{i}\right)=\tan ^{-1}\left(\operatorname{Im}\left\{F_{i}\right\} / \operatorname{Re}\left\{F_{i}\right\}\right)$ is the phase angle of $F_{i}$, and $\operatorname{Im}\left\{F_{i}\right\}$ and $\operatorname{Re}\left\{F_{i}\right\}$ are imaginary and real values of $F_{i}$, respectively. In the classification study, one-minute Oz EEG signals were recorded for each condition. There were 2985 input vectors obtained from each subjects, including the four gaze and one resting-state (non-gaze) conditions. In the application study, the beginning $0.5 \mathrm{~s}$ transition time (10 input vectors) in each trial (see Figure 2) was excluded to avoid the contamination of eye-motion artifact.

\section{Classification of SSVEP amplitude and phase features using multiclass SVM}

We adopted "one-against-all" multiclass SVM which constructs one binary SVM for each class to distinguish samples of one class from samples of all remaining classes [26,35-37].

In this study, the first-half (30 s) input vectors obtained from each condition (597 input vectors for each condition; 2985 input vectors in total) in the classification study were used as input data to train the multiclass SVM for each subject. The trained multiclass SVM was used to evaluate the classification performance in the classification study and to detect the gazed targets in the application study. The amplitude and phase features of training data were arranged into a $2 \times N(N=2985)$ matrix for the multiclass SVM training. The multiclass SVM was constructed by a set of binary SVMs to map input data into output space. The classification of multiclass SVM was done by finding the class with maximum output among all binary SVMs. It is worthy to notice that the multiclass SVM should be retrained at the beginning of each session due to the consideration of inter-session variability in SSVEPs.

\section{Determination of effective epoch for user's gaze condition}

Since SSVEP is time-locked and phase-locked signal contingent to the flickering timing of subject's gazed target [34], user's non-gaze condition will result in uncertainties in the detected SSVEP phases. SSVEPs induced from a phase-tagged visual stimulus have centralized phase distribution at the visual stimulation frequency, while resting-state $\mathrm{Oz}$ EEG signals usually have phase distribution, measured at the same frequency, randomly distributed over $0^{\circ}$ to $360^{\circ}$. Accordingly, the discrepancy of phase distribution can be used to discriminate gaze condition from non-gaze condition in the use of a 
phase-tagged SSVEP-based BCI. In this paper, Kolmogorov-Smirnov (K-S) test was adopted to collect sufficient SSVEP phase information for gaze-target detections. K-S test is a non-parametric method to determine if two sample sets are different from each other $[38,39]$. Therefore, we reasonably assume the resting-state phases, measured at the flickering frequency of visual stimulus, of Oz EEG signals were uniformly distributed over a full cycle. The effectiveness of gaze-target information for a sample set can be examined by checking whether its phase distribution is away from uniform distribution or not. The length of an effective epoch for gaze-target detection was initially set at $Z(Z=10)$ input vectors and sent to $\mathrm{K}-\mathrm{S}$ test to check its nonuniformness. If the data set could not reject the null hypothesis (i.e., $p$ value $>0.01$ ), one more input vector was added in the epoch. Once a data set passed K-S test, the data set was defined as an effective epoch. For each input vector in an effective epoch, a classification output was given by the trained multiclass SVM. The gazed target for each effective epoch was then identified using a plurality voting system by finding the class which gained a majority of votes. The overall flowchart of signal processing is shown in Figure 3.

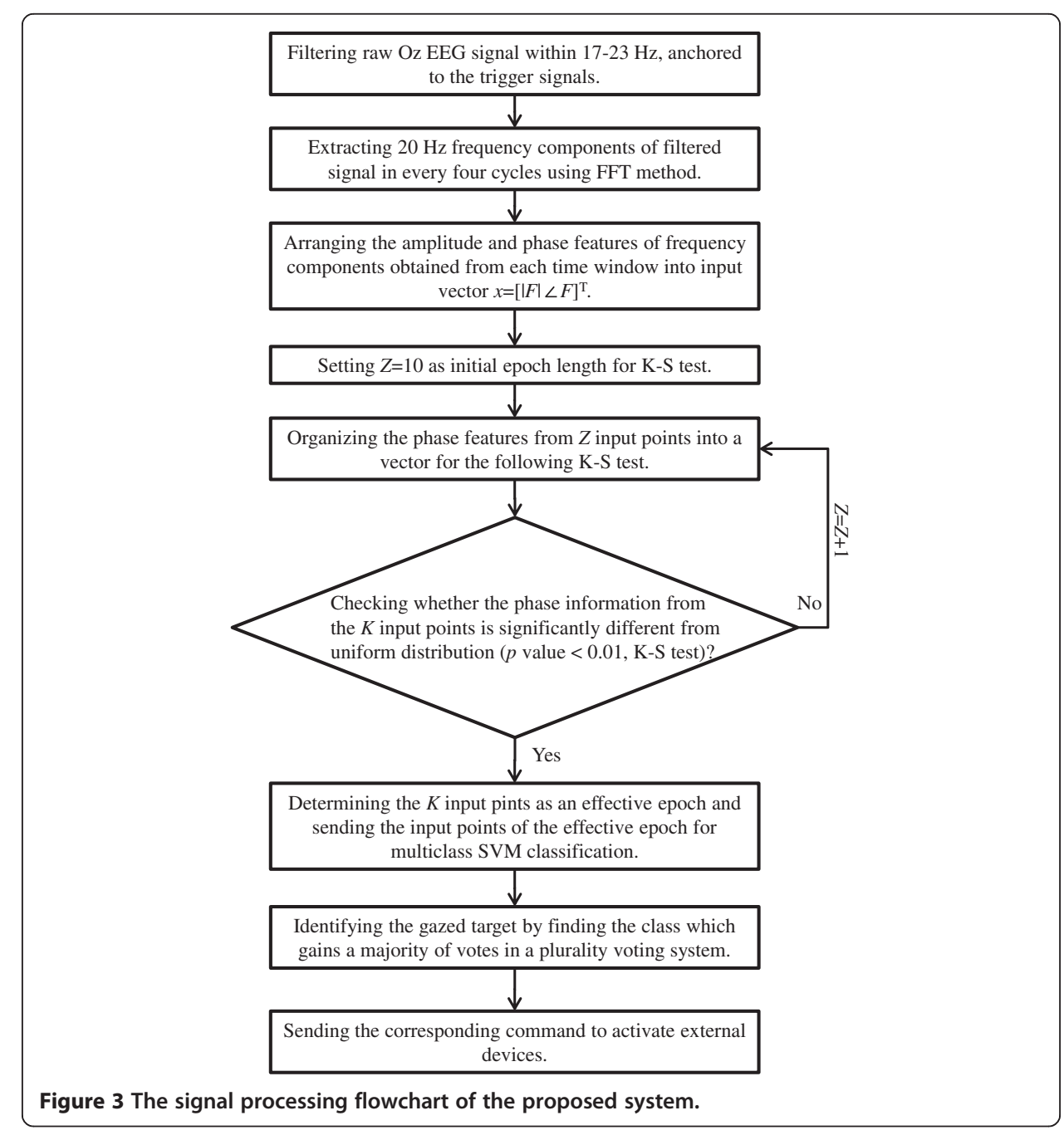




\section{Results}

Figure 4a shows the signal processing for extracting amplitude and phase features in subject 1 . The upper panel shows the raw Oz EEG signal from a 30-s recording and the lower panel presents the signal of raw Oz EEG signal filtered within 17-23 Hz. The flash onsets of LED flickering sequence, marked by red dashed vertical lines, were served as trigger events to segment the filtered EEG signal into cycles. The frequency components were extracted every 4 cycles with $75 \%$ overlapped sliding Hamming

(a)

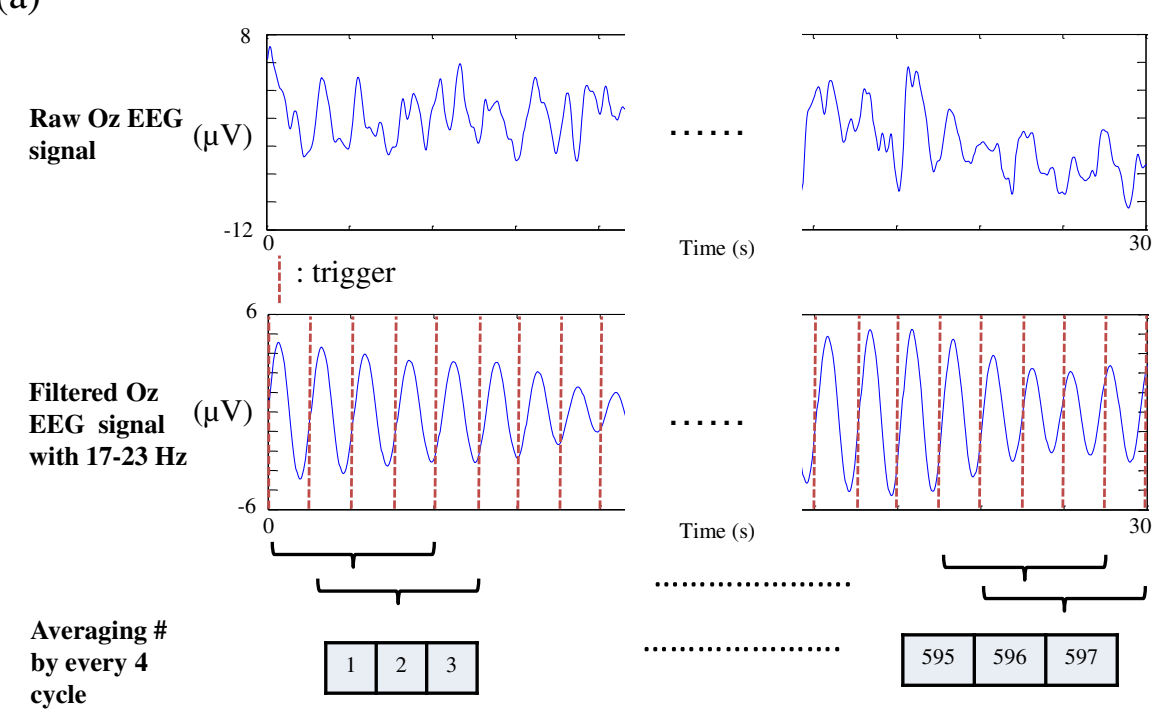

(b)
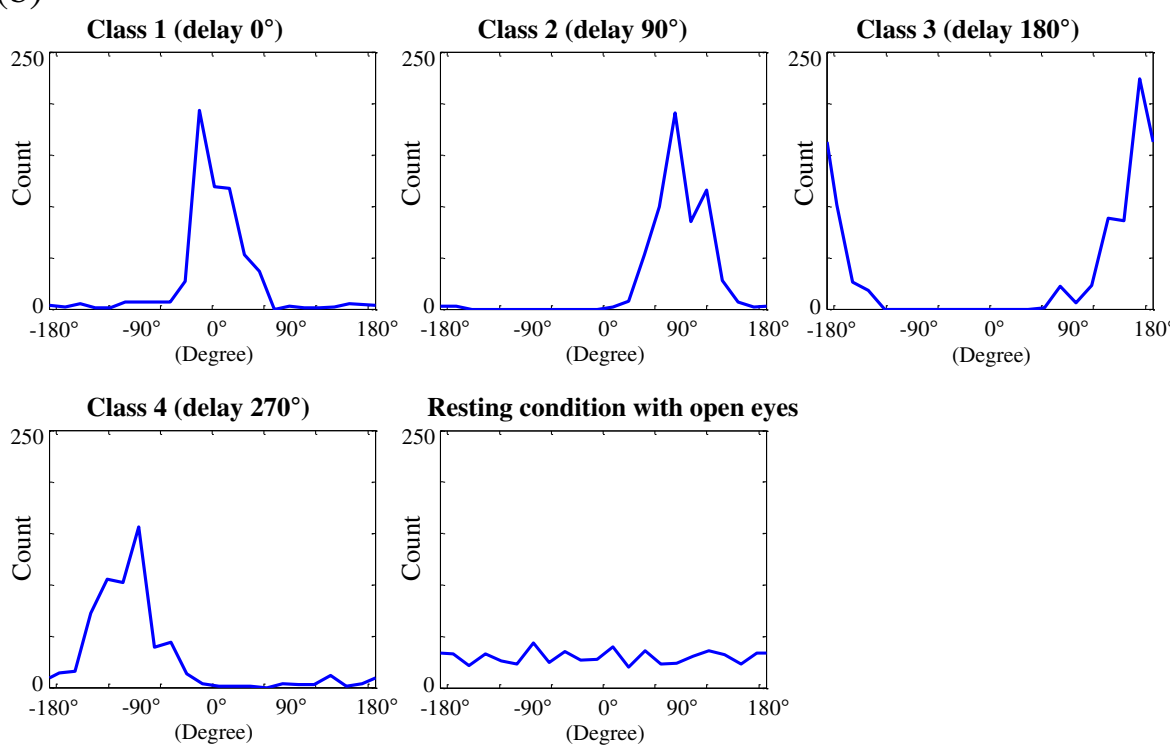

Figure 4 The signal processing for extracting frequency components and phase distributions obtained from the five gaze conditions. The signal processing for extracting amplitude and phase features in (a). Oz EEG signals were bandpass-filtered within 17-23 Hz to obtain SSVEP responses by means of applying a causal Butterworth filter (6th-order, IIR Butterworth filter). The frequency components of SSVEPs at $20 \mathrm{~Hz}$ were extracted in every 4 cycles using Fourier method with 75\% overlapped sliding Hamming window. The difference of phase distributions obtained from the four gaze conditions $\left(0^{\circ}, 90^{\circ}\right.$, $180^{\circ}, 270^{\circ}$ ) and one resting-state (non-gaze) condition in subject 1 (b). 
window, and the amplitude and phase features of frequency components were arranged into input vectors for multiclass SVM classifications. Figure $4 \mathrm{~b}$ demonstrates the difference of phase distributions obtained from the four gaze conditions $\left(0^{\circ}, 90^{\circ}, 180^{\circ}, 270^{\circ}\right)$ and one resting-state (non-gaze) condition in subject 1 . Each phase distribution was the phase features of frequency components gathered from 30-s recording in different condition. It can be observed that the phase distribution recorded from the restingstate condition (right-lower panel in Figure 4b) is a uniform distribution which can be distinguished from other gaze conditions using K-S test.

In our classification study, one half of the recorded data was used for multiclass SVM training, and the other half was used for multiclass SVM testing. Figure 5 shows the decision surfaces of trained multiclass SVMs in classifying the five classes (four gaze and one resting-state (non-gaze) conditions) in subject 1 and 6 . The solid lines are the decision boundaries to separate each class from others. Colors code the outputs of

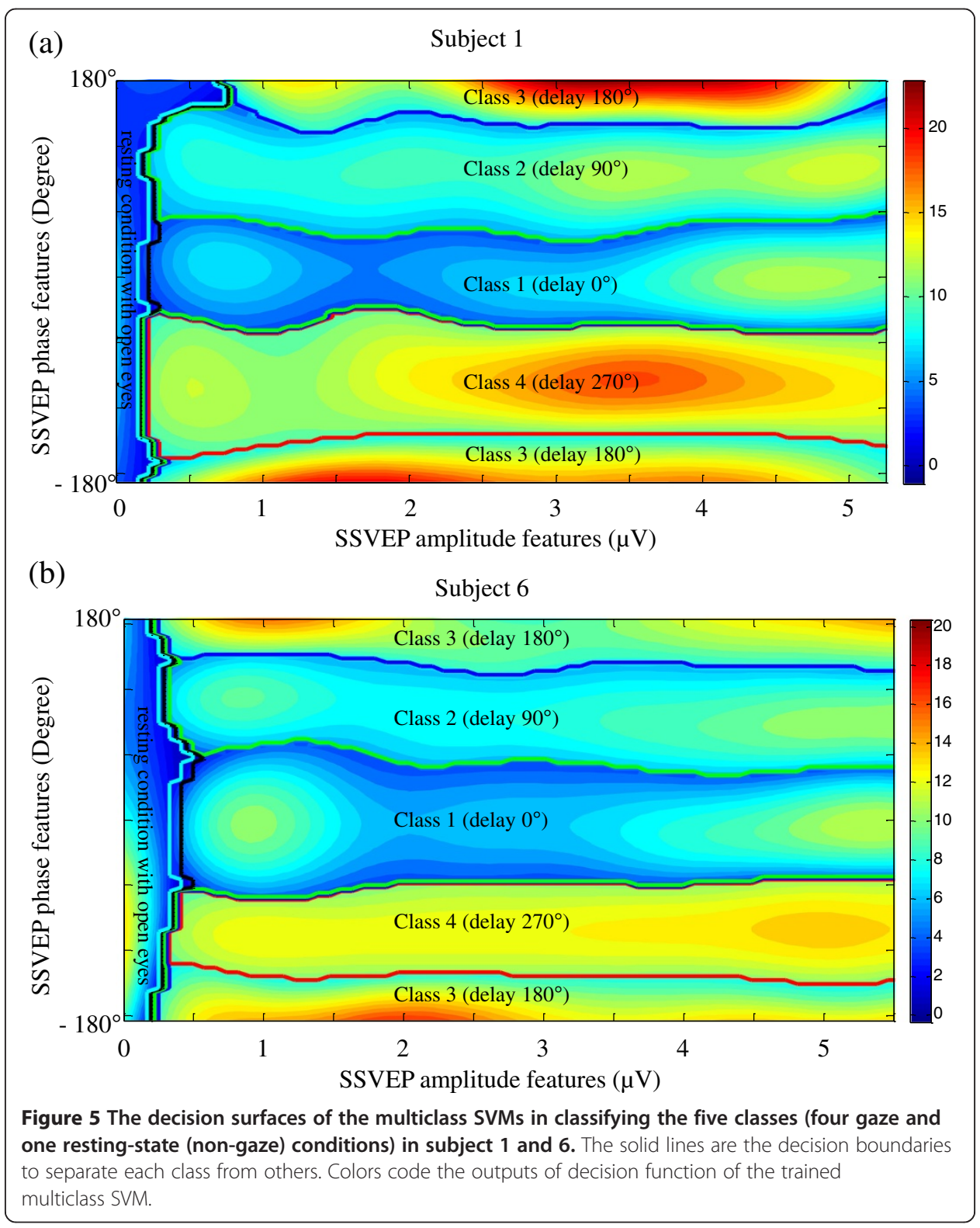


decision function of the trained multiclass SVM. The clear decision boundaries illustrate the feasibility of using the SSVEP amplitude and phase features in classifying different gaze conditions when operating a phase-tagged SSVEP-based BCI.

Table 2 presents the detected accuracies of multiclass SVM testing in the classification study. The averaged effective epoch lengths were from $0.59 \mathrm{~s}$ to $0.77 \mathrm{~s}$; the averaged accuracies were from $87.18 \%$ to $97.70 \%$; compared to the conventional epoch-average method with 14 cycles being averaged (detection interval $=0.7 \mathrm{~s}$ ) [2], the detection accuracies were from $60.71 \%$ to $82.62 \%$ for 20 subjects. The overall accuracies were $93.49 \%$ vs. $70.70 \%$ (multiclass SVM testing vs. the conventional epoch-average method) which demonstrates the superiority ( $p<0.01$, Wilcoxon signed rank test) of using multiclass SVM in improving the SSVEP-based BCI performance.

To quantify the on-line performance of multiclass SVM, a command transfer rate (CTI) and an information transfer rate (ITR) are introduced in addition to the accuracy. The CTI is defined as the total experimental time $\left(\mathrm{T}_{\text {total }}\right)$ divided by the number of total execution commands, and the ITR is defined as $[1,40]$ :

$$
\begin{aligned}
& \frac{\text { Bits }}{\text { command }}=\log _{2} S+P \log _{2} P+(1-P) \log _{2}[(1-P) /(S-1)], \\
& \text { ITR }=\frac{\text { Bits }}{\text { command }} \cdot \frac{60}{C T I},
\end{aligned}
$$

where $S$ is the total number of visual flickers $(S=4)$ and $P$ is the accuracy.

Table 3 shows the online results of execution time, accuracies, averaged effective epoch lengths and ITRs from twenty subjects in the application study. The accuracies were $90 \%, 97.5 \%, 91.25 \%, 83.75 \%, 93.75 \%$, 96.25\%, 88.75\%, 95\%, 88.75\%, 86.25\%, 92.5\%, $91.25 \%, 87.5 \%, 85 \%, 96.25 \%, 81.25 \%, 93.75 \%, 90 \%, 86.25 \%$, and $82.50 \%$; the averaged effective epoch lengths were $1.12 \pm 0.18,1.11 \pm 0.18,1.10 \pm 0.17,1.16 \pm 0.21,1.15 \pm 0.21$, $1.09 \pm 0.18,1.10 \pm 0.19,1.16 \pm 0.22,1.17 \pm 0.24,1.12 \pm 0.20,1.10 \pm 0.16,1.11 \pm 0.19,1.14 \pm$ $0.23,1.13 \pm 0.22,1.11 \pm 0.19,1.13 \pm 0.18,1.19 \pm 0.25,1.20 \pm 0.26,1.16 \pm 0.23$ and $1.11 \pm$ $0.17 \mathrm{~s}$; the ITRs were $50.87,66.72,53.73,39.73,56.73,64.68,49.38,59.14,47.34,44.49$, $56.28,53.27,45.98,42.31,63.80,37.07,55.51,48.41,43.52$, and $39.25 \mathrm{bits} / \mathrm{min}$ for subject 1 to subject 20, respectively. The averaged results (mean \pm SD) of overall twenty subjects showed accuracy rate was $89.88 \pm 4.76 \%$, averaged effective epoch length was $1.13 \pm 0.20 \mathrm{~s}$ and ITR was $50.91 \pm 8.70 \mathrm{bits} / \mathrm{min}$.

\section{Discussion}

Among the current assistive technologies, the $\mathrm{BCI}$ has drawn the greatest attention due to its independent of peripheral neuromuscular activities. A BCI recognizes the patterns of brain waves induced from elaborately designed task, and then translates the brain waves into control commands. However, since human brain is a complex system which usually exhibits remarkable inter-individual variability [12,41-44], therefore, choosing a robust classifier with flexibility to adapt inter-individual difference is crucial for an effective BCI. In this study, we adopted multiclass SVM to classify five conditions, including four gaze and one resting-state (non-gaze) conditions, in the use of a phase-tagged SSVEP-based BCI. The amplitude and phase information of SSVEPs were transformed into feature vectors for SVM training as well as classification. This is the 
Table 2 The detected accuracies of multiclass SVM testing and the conventional epochaverage method in the classification study

\begin{tabular}{|c|c|c|c|c|c|c|c|}
\hline \multirow{3}{*}{$\begin{array}{c}\text { Subject } \\
\text { index }\end{array}$} & \multicolumn{4}{|c|}{ Multiclass SVM-based method } & \multirow{3}{*}{$\begin{array}{c}\text { Averaged } \\
\text { accuracies } \\
\text { (Num }_{\text {correct }} / \\
\left.\text { Num }_{\text {total }}\right)\end{array}$} & \multirow{3}{*}{$\begin{array}{l}\text { Averaged } \\
\text { effective } \\
\text { epoch } \\
\text { lengths } \\
\text { (s) }\end{array}$} & \multirow{3}{*}{$\begin{array}{c}\text { The } \\
\text { conventional } \\
\text { epoch- } \\
\text { average } \\
\text { method } \\
\text { (detection } \\
\text { interval }=0.7 \\
\text { sec) }\end{array}$} \\
\hline & \multicolumn{4}{|c|}{ Accuracies for each class ( $\mathrm{Num}_{\text {correct }} / \mathrm{Num}_{\text {total }}$ ) } & & & \\
\hline & Class I & Class II & Class III & Class IV & & & \\
\hline \multirow[t]{2}{*}{1} & $97.62 \%$ & $98.18 \%$ & $94.23 \%$ & $94.55 \%$ & $96.08 \%$ & 0.59 & $82.62 \%$ \\
\hline & $(41 / 42)$ & $(54 / 55)$ & $(49 / 52)$ & $(52 / 55)$ & $(196 / 204)$ & & \\
\hline \multirow[t]{2}{*}{2} & $97.96 \%$ & $96.00 \%$ & $91.30 \%$ & $93.62 \%$ & $94.79 \%$ & 0.63 & $78.06 \%$ \\
\hline & $(48 / 49)$ & $(48 / 50)$ & $(42 / 46)$ & $(44 / 47)$ & $(182 / 192)$ & & \\
\hline \multirow[t]{2}{*}{3} & $97.56 \%$ & $88.68 \%$ & $90.38 \%$ & $91.49 \%$ & $91.71 \%$ & 0.62 & $65.82 \%$ \\
\hline & $(40 / 41)$ & $(47 / 53)$ & $(47 / 52)$ & $(43 / 47)$ & $(177 / 193)$ & & \\
\hline \multirow[t]{2}{*}{4} & $95.56 \%$ & $100 \%$ & $94.34 \%$ & $97.37 \%$ & $96.79 \%$ & 0.64 & $67.86 \%$ \\
\hline & $(43 / 45)$ & $(51 / 51)$ & $(50 / 53)$ & $(37 / 38)$ & $(181 / 187)$ & & \\
\hline \multirow[t]{2}{*}{5} & $94.59 \%$ & $92.59 \%$ & $91.38 \%$ & $93.62 \%$ & $92.86 \%$ & 0.61 & $77.38 \%$ \\
\hline & $(35 / 37)$ & $(50 / 54)$ & (53/58) & $(44 / 47)$ & $(182 / 196)$ & & \\
\hline \multirow[t]{2}{*}{6} & $94.87 \%$ & $97.50 \%$ & $95.45 \%$ & $93.02 \%$ & $95.18 \%$ & 0.72 & $64.29 \%$ \\
\hline & $(37 / 39)$ & $(39 / 40)$ & $(42 / 44)$ & $(40 / 43)$ & (158/166) & & \\
\hline \multirow[t]{2}{*}{7} & $91.43 \%$ & $93.48 \%$ & $97.73 \%$ & $93.35 \%$ & $94.64 \%$ & 0.71 & $75.00 \%$ \\
\hline & $(32 / 35)$ & $(43 / 46)$ & $(43 / 44)$ & $(41 / 43)$ & $(159 / 168)$ & & \\
\hline \multirow[t]{2}{*}{8} & $94.59 \%$ & $87.18 \%$ & $82.50 \%$ & 85.00 & $87.18 \%$ & 0.77 & $80.40 \%$ \\
\hline & $(35 / 37)$ & $(34 / 39)$ & $(33 / 40)$ & $(34 / 40)$ & $(136 / 156)$ & & \\
\hline \multirow[t]{2}{*}{9} & $91.49 \%$ & $96.15 \%$ & $93.02 \%$ & $97.83 \%$ & $94.68 \%$ & 0.64 & $70.92 \%$ \\
\hline & $(43 / 47)$ & $(50 / 52)$ & $(40 / 43)$ & $(45 / 46)$ & $(178 / 188)$ & & \\
\hline \multirow[t]{2}{*}{10} & $93.62 \%$ & $88.64 \%$ & $84.44 \%$ & $92.86 \%$ & $89.89 \%$ & 0.67 & $68.39 \%$ \\
\hline & $(44 / 47)$ & $(39 / 44)$ & $(38 / 45)$ & $(39 / 42)$ & $(160 / 178)$ & & \\
\hline \multirow[t]{2}{*}{11} & $89.13 \%$ & $90.48 \%$ & $95.00 \%$ & $87.50 \%$ & $90.34 \%$ & 0.68 & $66.33 \%$ \\
\hline & $(41 / 46)$ & $(38 / 42)$ & $(38 / 40)$ & $(42 / 48)$ & $(159 / 176)$ & & \\
\hline \multirow[t]{2}{*}{12} & $92.86 \%$ & $91.67 \%$ & $91.38 \%$ & $90.20 \%$ & $91.46 \%$ & 0.60 & $71.94 \%$ \\
\hline & $(39 / 42)$ & $(44 / 48)$ & $(53 / 58)$ & $(46 / 51)$ & (182/199) & & \\
\hline \multirow[t]{2}{*}{13} & $92.31 \%$ & $93.18 \%$ & $85.71 \%$ & $94.00 \%$ & $91.28 \%$ & 0.62 & $73.98 \%$ \\
\hline & $(48 / 52)$ & $(41 / 44)$ & $(42 / 49)$ & $(47 / 50)$ & (178/195) & & \\
\hline \multirow[t]{2}{*}{14} & $100 \%$ & $91.49 \%$ & $95.24 \%$ & $87.50 \%$ & $93.33 \%$ & 0.67 & $65.31 \%$ \\
\hline & $(43 / 43)$ & $(43 / 47)$ & $(40 / 42)$ & $(42 / 48)$ & $(168 / 180)$ & & \\
\hline \multirow[t]{2}{*}{15} & $95.56 \%$ & $89.80 \%$ & $98.11 \%$ & $100 \%$ & $95.92 \%$ & 0.61 & $63.78 \%$ \\
\hline & $(43 / 45)$ & $(44 / 49)$ & $(52 / 53)$ & $(49 / 49)$ & (188/196) & & \\
\hline \multirow[t]{2}{*}{16} & $95.35 \%$ & $98.00 \%$ & $100 \%$ & $97.50 \%$ & $97.70 \%$ & 0.69 & $68.88 \%$ \\
\hline & $(41 / 43)$ & $(49 / 50)$ & $(41 / 41)$ & $(39 / 40)$ & $(170 / 174)$ & & \\
\hline \multirow[t]{2}{*}{17} & $100 \%$ & $91.30 \%$ & $90.70 \%$ & $95.12 \%$ & $94.12 \%$ & 0.71 & $74.49 \%$ \\
\hline & $(40 / 40)$ & $(42 / 46)$ & $(39 / 43)$ & $(39 / 41)$ & $(160 / 170)$ & & \\
\hline \multirow[t]{2}{*}{18} & $93.62 \%$ & $100 \%$ & $91.11 \%$ & $87.76 \%$ & $92.90 \%$ & 0.66 & $72.96 \%$ \\
\hline & $(44 / 47)$ & $(42 / 42)$ & $(41 / 45)$ & $(43 / 49)$ & $(170 / 183)$ & & \\
\hline \multirow[t]{2}{*}{19} & $93.33 \%$ & $92.45 \%$ & $93.33 \%$ & $97.67 \%$ & $94.09 \%$ & 0.65 & $60.71 \%$ \\
\hline & $(42 / 45)$ & $(49 / 53)$ & $(42 / 45)$ & $(42 / 43)$ & $(175 / 186)$ & & \\
\hline
\end{tabular}


Table 2 The detected accuracies of multiclass SVM testing and the conventional epochaverage method in the classification study (Continued)

\begin{tabular}{lrrrrrrc}
\hline 20 & $98.08 \%$ & $91.11 \%$ & $91.84 \%$ & $97.78 \%$ & $94.76 \%$ & 0.63 & $64.80 \%$ \\
& $(51 / 52)$ & $(41 / 42)$ & $(45 / 49)$ & $(44 / 45)$ & $(181 / 191)$ & & \\
& & & & & \\
& Average $($ mean \pm SD) & & $93.49 \pm 2.58 \%$ & $0.66 \pm 0.05$ & $70.70 \pm 6.06 \%$ \\
\hline
\end{tabular}

first SVM study on the classification of phase-tagged SSVEP-based BCIs. The study results have shown the feasibility of the proposed system, which demonstrates an effective classifier could play an important role in designing a reliable BCI.

It has been mentioned several times in the literature that the amplitude of SSVEP can be used to discriminate the evoked SSVEP from background physiological activities. For example, Wu et al. [45] defined an SSVEP signal-to-noise ratio (SNR) in which comparing the SSVEP power divided by averaged power of the selected frequency window. Midderdorf et al. [46] applied an amplitude threshold for SSVEP to control a binary switch. However, it is difficult to judge subject's gaze condition only from the information of SSVEP amplitude, owing to each subject has his/her own SSVEP amplitude-frequency characteristic which results in the SSVEP responses varying with different visual stimulation frequencies. Moreover, the inter-subject variation in background physiological activities also makes uncertainties in discriminating gaze condition from non-gaze condition if only SSVEP amplitude is used.

Table 3 The online results of execution time, accuracies, averaged effective epoch lengths and ITRs from twenty subjects in the application study

\begin{tabular}{|c|c|c|c|c|}
\hline $\begin{array}{l}\text { Subject } \\
\text { index }\end{array}$ & $\mathrm{T}_{\text {total }}(\mathrm{s})$ & $\begin{array}{l}\text { Accuracy }\left(\text { Num }_{\text {correct }} /\right. \\
\left.\text { Num }_{\text {total }}\right)\end{array}$ & $\begin{array}{l}\text { Averaged effective epoch } \\
\text { lengths (s) }\end{array}$ & $\begin{array}{c}\text { ITR } \\
\text { (bits/min) }\end{array}$ \\
\hline 1 & 129.50 & $90.00 \%(72 / 80)$ & $1.12 \pm 0.18$ & 50.87 \\
\hline 2 & 128.90 & $97.50 \%(78 / 80)$ & $1.11 \pm 0.18$ & 66.72 \\
\hline 3 & 128.05 & $91.25 \%(73 / 80)$ & $1.10 \pm 0.17$ & 53.73 \\
\hline 4 & 133.15 & $83.75 \%(67 / 80)$ & $1.16 \pm 0.21$ & 39.73 \\
\hline 5 & 132.30 & $93.75 \%(75 / 80)$ & $1.15 \pm 0.21$ & 56.73 \\
\hline 6 & 126.90 & $96.25 \%(77 / 80)$ & $1.09 \pm 0.18$ & 64.68 \\
\hline 7 & 127.75 & $88.75 \%(71 / 80)$ & $1.10 \pm 0.19$ & 49.38 \\
\hline 8 & 132.65 & $95.00 \%(76 / 80)$ & $1.16 \pm 0.22$ & 59.14 \\
\hline 9 & 133.25 & $88.75 \%(71 / 80)$ & $1.17 \pm 0.24$ & 47.34 \\
\hline 10 & 129.95 & $86.25 \%(69 / 80)$ & $1.12 \pm 0.20$ & 44.49 \\
\hline 11 & 127.65 & $92.50 \%(74 / 80)$ & $1.10 \pm 0.16$ & 56.28 \\
\hline 12 & 129.15 & $91.25 \%(73 / 80)$ & $1.11 \pm 0.19$ & 53.27 \\
\hline 13 & 131.35 & $87.50 \%(70 / 80)$ & $1.14 \pm 0.23$ & 45.98 \\
\hline 14 & 130.75 & $85.00 \%(68 / 80)$ & $1.13 \pm 0.22$ & 42.31 \\
\hline 15 & 128.65 & $96.25 \%(77 / 80)$ & $1.11 \pm 0.19$ & 63.80 \\
\hline 16 & 130.35 & $81.25 \%(65 / 80)$ & $1.13 \pm 0.18$ & 37.07 \\
\hline 17 & 135.20 & $93.75 \%(75 / 80)$ & $1.19 \pm 0.25$ & 55.51 \\
\hline 18 & 136.10 & $90.00 \%(72 / 80)$ & $1.20 \pm 0.26$ & 48.41 \\
\hline 19 & 132.85 & $86.25 \%(69 / 80)$ & $1.16 \pm 0.23$ & 43.52 \\
\hline 20 & 128.85 & $82.50 \%(66 / 80)$ & $1.11 \pm 0.17$ & 39.25 \\
\hline $\begin{array}{c}\text { Average } \\
(\text { mean } \pm \text { SD) }\end{array}$ & $130.67 \pm 2.60$ & $89.88 \pm 4.76 \%$ & $1.13 \pm 0.20$ & $50.91 \pm 8.70$ \\
\hline
\end{tabular}


Accordingly, in addition to SSVEP amplitude, we also took the information of SSVEP phase into account. Due to the advantages of time-locked and phase-locked characteristics of SSVEP, the SSVEPs generated from the subject's gaze condition usually result in a centralized phase distribution. In contrast, the EEG phases obtained from subject's resting-state (non-gaze) condition are irrelevant to flickering onsets of visual stimuli. Figure 6 shows the $20 \mathrm{~Hz}$ phases of Oz EEG signals obtained from the gaze and the resting-state (non-gaze) conditions in twenty subjects. The EEG phases were calibrated to the mean phase of SSVEP phases from gazing at $\operatorname{LED}_{1}\left(\theta_{1}=0^{\circ}\right)$ in each subject for the purpose of cross-subject comparison. Figure $6 \mathrm{a}-\mathrm{e}$ show the calibrated phases of Oz EEG signals at $20 \mathrm{~Hz}$ when subjects were gazing at $\mathrm{LED}_{1}, \mathrm{LED}_{2}, \mathrm{LED}_{3}, \mathrm{LED}_{4}$, and resting-state (non-gaze) condition for thirty-second recordings (597 fast Fourier transform (FFT) time windows in each subject, FFT window length $=200 \mathrm{~ms}$, window overlapping $=75 \%$; twenty subjects pooled), respectively. The solid lines show the mean phases, while the dashed lines indicate the mean plus/minus the standard deviations (i.e., mean $\pm \mathrm{SD}$ ) over the twenty subjects. The phase distributions obtained from the four gaze conditions (Figure $6 \mathrm{a}-\mathrm{d}$ ) showed significant differences with uniform distributions $(p<0.001, \mathrm{~K}-\mathrm{S}$ test). The phase distribution checked by K-S test showed no statistical difference with uniform distribution $(p>0.05)$ in Figure 6e. It echoes the decision surface of multiclass SVM results in Figure 5, in which the non-gaze class presented a uniform distribution spanning over 0 degree to 360 degree with low amplitude at stimulation frequency. It also endorses the feasibility of using phase distribution to discriminate gaze condition from non-gaze condition.

Since brain waves are dynamic activities which are contingent upon variations in a subject's performance and state, linked to fluctuations in expectation, attention, arousal, and task strategy [47-52], the generation of command output from an EEG interval involving SSVEP-unrelated information may sometimes cause unexpected pitfalls in BCI operation. To prevent the outputs of trained multiclass SVM from oversensitivity to input data, the present study generated command outputs from effective epochs. An effective epoch was determined to define the length of input data, containing sufficient SSVEP information for a valid BCI output, by checking the phase distribution of input points using K-S test. The input vectors in a determined effective epoch were sequentially inputted into a trained multiclass SVM in each subject, and the multiclass SVM outputs generated from those input vectors were gathered to produce a valid output through a plurality voting process. The plurality voting process determined the valid output by finding the visual target which gained a majority of votes from the multiclass SVM outputs of all input points in the effective epoch, so that the accuracy and specificity of the proposed BCI could be improved.

In our previous publication [2], subject's gazed target was identified using the phase feature of SSVEP only. Besides, the SSVEP was averaged over a large amount of epochs ( 30 epochs) to increase SNR and the confidence of phase detection. The epochaverage process caused a long detection time (4.83 s/command) and resulted in the consequence of low ITR. In contrast, in this multiclass SVM study, both the amplitude and phase features of SSVEP were used and extracted in every 4 cycles. Input data (amplitude and phase features of SSVEP) were mapped into high-dimensional feature space by SVM kernel to achieve better classification. Owing to the input of dual features and the benefit of multiclass SVM in classification, the averaged detection time 

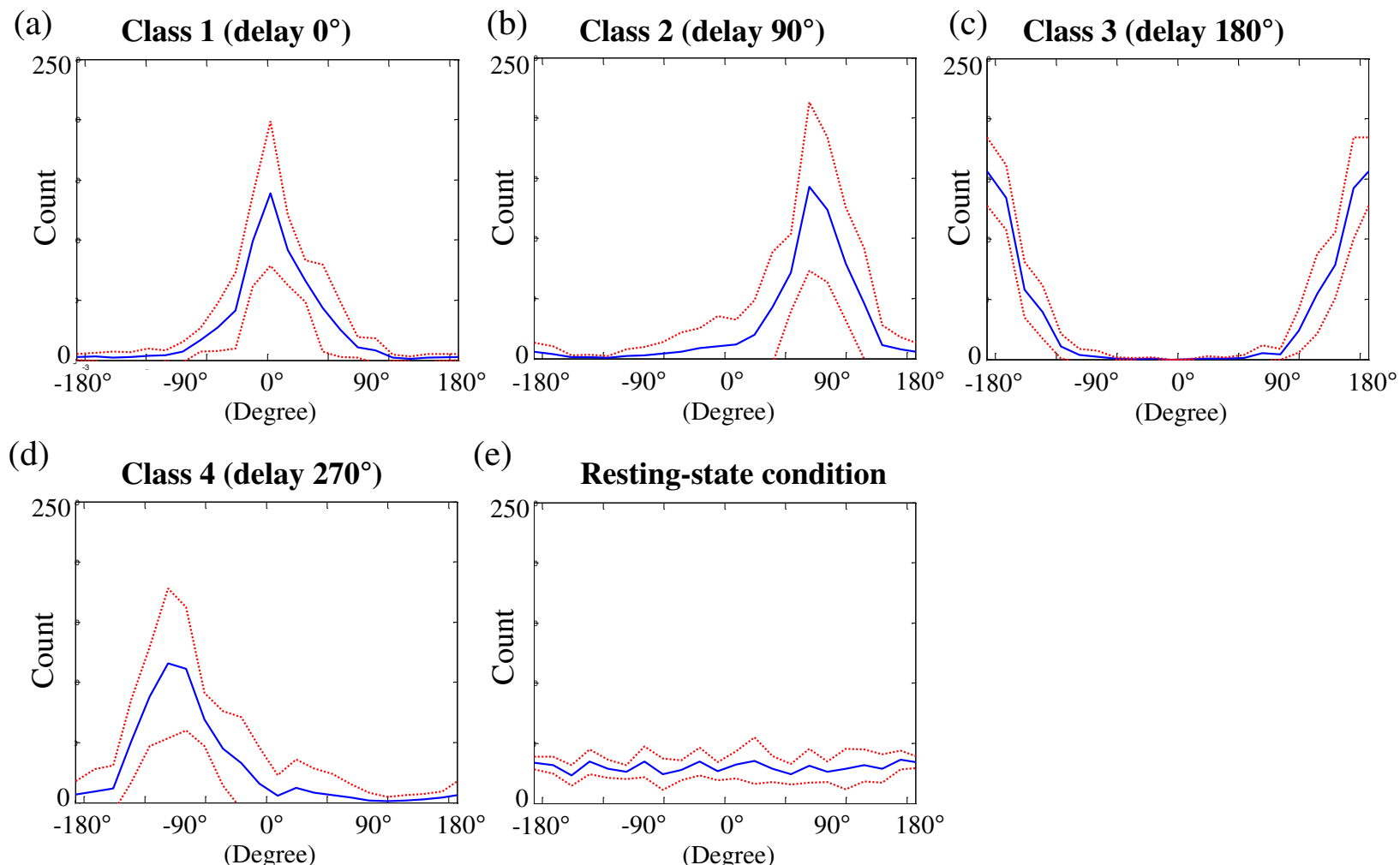

(e)

Resting-state condition

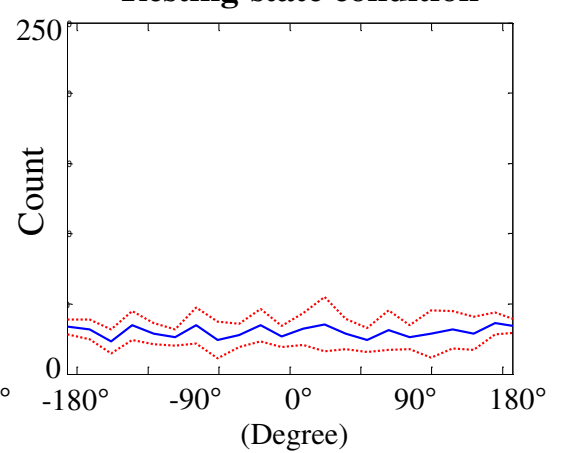

Figure 6 The phase distribution of SSVEPs at $20 \mathrm{~Hz}$ obtained from the gaze and the resting-state (non-gaze) conditions in twenty subjects using Fourier method. The phase distributions obtained from the four gaze conditions ( $\mathbf{a}-\mathbf{d})$ showed significant differences with uniform distributions $(p<0.001$, Kolmogorov-Smirnov (K-S) test), while the phase distribution checked by K-S test showed no statistical difference with uniform distribution $(p>0.05)$ in $(\mathbf{e})$. 
$\left(\mathrm{T}_{\text {total }} / \mathrm{Num}_{\text {total }}\right.$ ) in our application study (see Table 3 ) is $1.63 \mathrm{~s} /$ command which is greatly shorter than the detection time in our previous study.

The present study focuses on testing the capability of using multiclass SVM to identify subject's gazed targets by classifying amplitude and phase features of SSVEPs. However, according to some previous literatures, the phase of SSVEP can be influenced by the jitter of stimulator triggers [15,17], strategic planning, organized searching [53], memory load [54], emotional arousal [55], adaption of long-term stimulation [56], etc. Training a new classifier has to be learned at the beginning of each session or adopting on-line phase recalibration methods may be possible solutions to account for this phase drift problem in our current design. The issue of recalibrating on-line SSVEP phase can be referred to our previous publication [43] which utilized a biphasic stimulation approach to account for the phase drift of SSVEP during flicker stimulation. Our future work will combine this multiclass SVM method with on-line phase re-calibration technique to improve its applicability.

\section{Conclusions}

In this paper, a multiclass SVM-based classification approach is proposed to discriminate subjects' gazed targets for communication purposes. Subjects shifted their gazes at different visual stimuli, and the amplitude and phase information of the induced SSVEP were extracted for multiclass SVM to recognize the gazed targets. The salient features of the proposed system are: (1) the amplitude and phase features were used to classify four gaze and one resting-state (non-gaze) conditions; (2) the effective epoch was determined to define the length of input data, containing sufficient SSVEP information for a valid BCI output; (3) SVM outputs generated from input vectors of effective epochs were gathered to produce valid outputs through a plurality voting process. The system demonstrates its feasibility with ITR performance in healthy subjects. In future studies, the efficiency and reliability of this system for patients with motor neuron diseases should be further investigated.

Competing interests

The authors declare that they have no competing interests.

Authors' contributions

CLY carried out the EEG data analysis by SVM and drafted the manuscript. WMC, CYC, YTW and GYL participated in the design of the study and performed the statistical analysis. PLL supervised the study, helped drafting and revising the manuscript. All authors read and approved the final manuscript.

\section{Acknowledgements}

This study was funded by the National Central University, the Center for Dynamical Biomarkers and Translational Medicine, National Science Council (99-2628-E-008-003, 99-2628-E-008-012, 100-2628-E-008-001, 100-2221-E -008-006, 100-2623-E-008-006-D), Center for Dynamical Biomarkers and Translational Medicine, National Central University, Taiwan (NSC 100-2911-I-008-001), Veterans General Hospital University System of Taiwan Joint Research Program (VGHUST101-G4-2-2), Taoyuan General Hospital Intramural Project (PTH10116), Cheng Hsin General Hospital Intramural Project ((298)101-06), Cheng Hsin and Yang-Ming University Program (100F117CY30), National Taiwan Normal University (NSC 98-2511-S-003-050-MY3), and National Central University and Landseed Hospital Joint Research Program (NCU-LSH-101-A-023).

\section{Author details}

${ }^{1}$ Department of Electrical Engineering, National Central University, Jhongli, Taiwan. ${ }^{2}$ Laboratory of Integrated Brain Research, Department of Medical Research and Education, Taipei Veterans General Hospital, Taipei, Taiwan. ${ }^{3}$ Institute of Brain Science, National Yang-Ming University, Taipei, Taiwan. ${ }^{4}$ Institute of Computer Science and Information Engineering, National Ilan University, Ilan, Taiwan. ${ }^{5}$ Science Education Center, National Taiwan Normal University, Taipei, Taiwan. ${ }^{6}$ Department of Biomedical Imaging and Radiological Sciences, National Yang-Ming University, Taipei, Taiwan. ${ }^{7}$ Department of Medical Image, Cheng Hsin General Hospital, Taipei, Taiwan. 


\section{References}

1. Wolpaw JR, Birbaumer N, McFarland DJ, Pfurtscheller G, Vaughan TM: Brain-computer interfaces for communication and control. Clin Neurophysiol 2002, 113:767-791.

2. Lee PL, Sie JJ, Liu YJ, Lee MH, Shu CH, Li PH, Sun CW, Shyu KK: An SSVEP-actuated brain computer interface using phase-tagged flickering sequences: a cursor system. Ann Biome Eng 2010, 38:2383-2397.

3. Parini S, Maggi L, Turconi AC, Andreoni G: A robust and self-paced BCl system based on a four class SSVEP paradigm: algorithms and protocols for a high-transfer-rate direct brain communication. Comput Intell Neurosci 2009, 2009:11.

4. Zhu D, Molina G, Mihajlovic V, Aarts R: Phase synchrony analysis for SSVEP-based BCls. Proc 2nd Int Conf Comput Eng Technol 2010, 2:329-333.

5. Herrmann CS: Human EEG responses to $1-100 \mathrm{~Hz}$ flicker: Resonance phenomena in visual cortex and their potential correlation to cognitive phenomena. Exp Brain Res 2001, 137:346-353.

6. Pastor MA, Artieda J, Arbizu J, Valencia M, Masdeu JC: Human cerebral activation during steady-state visualevoked responses. J Neurosci 2003, 23:11621-11627.

7. Vialatte FB, Maurice M, Dauwels J, Cichocki A: Steady-state visually evoked potentials: Focus on essential paradigms and future perspectives. Prog Neurobiol 2010, 90:418-438.

8. Cheng M, Gao X, Gao S: Design and implementation of a brain-computer interface with high transfer rate. IEEE Trans Biomed Eng 2002, 49:1181-1186.

9. Wang Y, Wang R, Gao X, Hong B, Gao S: A practical VEP-based brain-computer interface. IEEE Trans Neural Syst Rehab Eng 2006, 14:234-239.

10. Wu Z, Yao D: Frequency detection with stability coefficient for steady-state visual evoked potential (SSVEP)based BCls. J Neural Eng 2008, 5:36-43.

11. Lee PL, Yeh CL, Cheng JYS, Yang CY, Lan GY: An SSVEP-based BCl using high duty-cycle visual flicker. IEEE Trans Biomed Eng 2011, 58:3350-3359.

12. Jia C, Gao X, Hong B, Gao S: Frequency and phase mixed coding in SSVEP-based brain-computer interface. IEEE Trans. Biomed. Eng. 2011, 58:200-206.

13. Shyu K, Chiu YJ, Lee PL, Lee MH, Sie JJ, Wu CH, Wu YT, Tung PC: Total Design of an FPGA-Based Brain Computer Interface Control Hospital Bed Nursing System. IEEE Trans Industrial Electronics. in press.

14. Chang HC, Lee PL, Lo MT, Lee IH, Yeh TK, Chang CY: Independence of Amplitude-Frequency and Phase Calibrations in an SSVEP-Based BCI Using Stepping Delay Flickering Sequences. IEEE Trans Neural Syst Rehabil Eng 2012, 20(3):305-312.

15. Wolpaw JR: Brain-Computer Interfaces Principles and Practice. In Edited by Wolpaw EW USA, Wolpaw EW. USA: Oxford University Press; 2012:165-187.

16. Lopez-Gordo MA, Prieto A, Pelayo F, Morillas C: Use of phase in brain-computer interfaces based on steadystate visual evoked potentials. Neural Processing Lett. 2010, 32:1-9.

17. Wong CM, Wang B, Wan F, Mak PU, Mak PI, Vai Ml: An improved phase-tagged stimuli generation method in steady-state visual evoked potential based brain-computer interface. Proc 3rd Int Conf Biomed Eng Informat 2010, 2:745-749.

18. Bakardjian $H$, Tanaka T, Cichocki A: Emotional faces boost up steady-state visual responses for brain-computer interface. Neuroreport 2011, 22:121-125.

19. Morgan ST, Hansen JC, Hillyard SA: Selective attention to stimulus location modulates the steady-state visual evoked potential. Proc Nat Acad Sci USA 1996, 93:4770-4774.

20. Thompson JC, Tzambazis K, Stough C, Nagata K, Silberstein RB: The effects of nicotine on the $13 \mathrm{~Hz}$ steady-state visually evoked potential. Clin Neurophysiol 2000, 111:1589-1595.

21. Gray M, Kemp AH, Silberstein RJ, Nathan PJ: Cortical neurophysiology of anticipatory anxiety: an investigation utilizing steady state probe topography (SSPT). Neuroimage 2003, 20:975-986.

22. Boser B, Guyon I, Vapnik VN: A training algorithm for optimal margin classifiers. In Proceedings of the 5 th Annual ACM Workshop on Computational Learning Theory. 1992:144-152.

23. Cortes C, Vapnik V: Support-vector networks. Machine Learning 1995, 20:273-297.

24. Drucker H, Burges CJC, Kaufman L, Smola AJ, Vapnik V: Support vector regression machines. In Advances in Neural Information Processing Systems. Edited by Mozer M, Jordan M, Petsche T. Cambridge, MA: MIT Press; 1997:155-161.

25. Vapnik V: Statistical learning theory. New York: Wiley; 1998.

26. Burges CJC: A tutorial on support vector machines for pattern recognition. In Data Mining and Knowledge Discovery 2. Hingham, MA, USA: Kluwer Academic Publishers; 1998:121-167.

27. Banlz V, Schölkopf B, Bülthoff HH, Burges C, Vapnik V, Vetter T: Comparison of view based object recognition algorithms using realistic 3D models. In Proc. ICANN. Edited by Malsburg C, Seelen W, Vorbrüggen JC, Sendhoff B. Berlin, Germany; 1996:251-256.

28. Schmidt M: Identifying speaker with support vector networks. Interface, Sydney, Australia: Proc; 1996.

29. Joachims T: Text categorization with support vector machines: Learning with many relevant features. Technical Report 23, University at Dortmund, LS VIII; 1997.

30. Allwein $E L$, Schapire RE, Singer $Y$ : Reducing multiclass to binary: a unifying approach for margin classifiers. J. Machine Learning Research 2000, 1:113-141.

31. Wang $X$, Paliwal KK: Feature extraction and dimensionality reduction algorithms and their applications in vowel recognition. Pattern Recognition 2003, 36:2129-2139.

32. Rifkin R, Klautau A: In defence of one-vs-all classification. J. Machine Learning Research 2004, 5:101-141.

33. Liu S, Yi H, Chia LT, Rajan D: Adaptive hierarchical multiclass SVM classifier for texture-based image classification. Pro. of ICME 2005:1190-1193. 
34. Spehlmann R: Evoked potential primer. Boston, America: Butterworth Press; 1985.

35. Weston J, Watkins C: Multi-class support vector machines. Technical Report CSDTR-98-04. England: Department of Computer Science, Royal Holloway, University of London; 1998.

36. Furey TS, Cristianini N, Duffy N, Bednarski DW, Schummer M, Haussler D: Support vector machine classification and validation of cancer tissue samples using microarray expression data. Bioinformatics 2000, 16:906-914.

37. Cristianini N, Shawe-Taylor J: An introduction to support vector machines and other kernel-based learning methods. UK: Cambridge University Press; 2000.

38. Conover W: Practical nonparametric statistics. New York, NY: John Wiley and Sons; 1980.

39. Press WH, Flannery BP, Teukolsky SA, Vetterling WT: Numerical recipes in C. Cambridge University Press; 1992.

40. Kelly SP, Lalor EC, Reilly RB, Foxe JJ: Visual spatial attention tracking using high density SSVEP data for independent brain-computer communication. IEEE Trans Neural Syst Rehabil Eng 2005, 13:172-178.

41. Brunner C, Naeem M, Leeb R, Graimann B, Pfurtscheller G: Spatial filtering and selection of optimized components in four class motor imagery EEG data using independent components analysis. Pattern Recognition Lett 2007, 28:957-964.

42. Yeh $\mathrm{CL}$, Chang HC, Wu CH, Lee PL: Extraction of single-trial cortical beta oscillatory activities in EEG signals using empirical mode decomposition. Biomed Eng Online 2010, 9:25.

43. Wu HY, Lee PL, Chang HC, Hsieh JC: Accounting for phase drifts in SSVEP-based BCls by means of biphasic stimulation. IEEE Trans. Biomed. Eng. 2011, 58:1394-1402.

44. Ang KK, Chin ZY, Zhang H, Guan C: Mutual information-based selection of optimal spatial-temporal patterns for single-trial EEG-based BCls. Pattern Recognition 2012, 45:2137-2144.

45. Wu Z, Lai Y, Xia Y, Wu D, Yao D: Stimulator selection in SSVEP-based BCI. Med Eng Phys 2008, 30:1079-1088

46. Middendorf M, McMillan G, Calhoun G, Jones KS: Brain-computer interfaces based on the steady-state visualevoked response. IEEE Trans Rehabil Eng 2000, 8:211-214.

47. Earle JB: Task difficulty and EEG alpha asymmetry: an amplitude and frequency analysis. Neuropsychobiology 1998, 20:95-112.

48. Hoffman RE, Buchsbaum MS, Escobar MD, Makuch RW, Nuechterlein KH, Guich SM: EEG coherence of prefrontal areas in normal and schizophrenic males during perceptual activation. J. Neuropsychiatry and Clin. Neurosciences 1991, 3:169-175.

49. Yabe H, Satio F, Fukushima Y: Median method for detecting endogenous event-related brain potentials. Electroencephalography and Clin. Neurophysiol. 1993, 87:403-407.

50. Haig AR, Gordon E, Rogers G, Anderson J: Classification of single-trial ERP sub-types: application of globally optimal vector quantization using simulated annealing. Electroencephalography and Clin. Neurophysiol. 1995, 94:288-297.

51. Bastiaansen MCM, Bocker KBE, Cluitmans PJM, Brunia CHM: Event-related desynchronization related to the anticipation of a stimulus providing knowledge of results. Clin Neurophysiol 1999, 110:250-260.

52. Bastiaansen MCM, Bocker KBE, Brunia CHM: Event-related desynchronization during anticipatory attention for an upcoming stimulus: a comparative EEG/MEG study. Clin Neurophysiol 2001, 112:393-403.

53. Silberstein RB, Ciorciari J, Pipingas A: Steady-state visually evoked potential topography during the Wisconsin card sorting test. Electroencephalogr Clin Neurophysiol 1995, 96(1):24-35.

54. Silberstein RB, Nunez PL, Pipingas A, Harris P, Danieli F: Steady state visually evoked potential (SSVEP) topography in a graded working memory task. Int J Psychophysiol 2001, 42(2):219-232.

55. Keil A, Gruber T, Muller MM, Moratti S, Stolarova M, Bradley MM, Lang PJ: Early modulation of visual perception by emotional arousal: evidence from steady-state visual evoked brain potentials. Cogn Affect Behav Neurosci 2003, 3(3):195-206

56. Bergholz R, Lehmann TN, Fritz G, Ruther K: Fourier transformed steady-state flash evoked potentials for continuous monitoring of visual pathway function. Doc Ophthalmol 2008, 116(3):217-229.

doi:10.1186/1475-925X-12-46

Cite this article as: Yeh et al:: Improvement of classification accuracy in a phase-tagged steady-state visual evoked potential-based brain computer interface using multiclass support vector machine. BioMedical Engineering OnLine 2013 12:46.

\section{Submit your next manuscript to BioMed Central and take full advantage of:}

- Convenient online submission

- Thorough peer review

- No space constraints or color figure charges

- Immediate publication on acceptance

- Inclusion in PubMed, CAS, Scopus and Google Scholar

- Research which is freely available for redistribution 